\title{
Labdane diterpenes protect against anoxia/ reperfusion injury in cardiomyocytes: involvement of AKT activation
}

\author{
I Cuadrado ${ }^{1}$, M Fernández-Velasco², L Boscáa ${ }^{\star 2}$ and B de las Heras ${ }^{\star, 1}$
}

Several labdane diterpenes exert anti-inflammatory and cytoprotective actions; therefore, we have investigated whether these molecules protect cardiomyocytes in an anoxia/reperfusion (A/R) model, establishing the molecular mechanisms involved in the process. The cardioprotective activity of three diterpenes (T1, T2 and T3) was studied in the H9c2 cell line and in isolated rat cardiomyocyte subjected to A/R injury. In both cases, treatment with diterpenes $T 1$ and T2 protected from A/R-induced apoptosis, as deduced by a decrease in the percentage of apoptotic and caspase-3 active positive cells, a decrease in the $\mathrm{Bcl}-2 / \mathrm{Bax}$ ratio and an increase in the expression of antiapoptotic proteins. Analysis of cell survival signaling pathways showed that diterpenes $T 1$ and T2 added after A/R increased phospho-AKT and phospho-ERK 1/2 levels. These cardioprotective effects were lost when AKT activity was pharmacologically inhibited. Moreover, the labdane-induced cardioprotection involves activation of AMPK, suggesting a role for energy homeostasis in their mechanism of action. Labdane diterpenes (T1 and T2) also exerted cardioprotective effects against A/R-induced injury in isolated cardiomyocytes and the mechanisms involved activation of specific survival signals (PI3K/AKT pathways, ERK1/2 and AMPK) and inhibition of apoptosis.

Cell Death and Disease (2011) 2, e229; doi:10.1038/cddis.2011.113; published online 10 November 2011

Subject Category: Experimental Medicine

Cardiac injury due to ischemia (anoxia)/reperfusion $(I(A) / R)$ is caused by two main mechanisms, apoptosis and necrosis, the latter being accelerated by reperfusion. ${ }^{1-5}$ Knowledge of molecular mechanisms involved in A/R reveals that cardioprotection is afforded by activation during reperfusion of the anti-apoptotic and prosurvival kinase signaling cascades PI3K-AKT and p42/p44 extracellular signal-regulated kinases (ERK1/2). ${ }^{6,7}$ Early activation of ERK1/2 inhibits procaspase processing and induces the expression of proteins of the antiapoptotic $\mathrm{Bcl}-2$ family ( $\mathrm{Bcl}-2$ and $\mathrm{Bcl}-\mathrm{xL})$. Thus, therapeutic strategies that target and attenuate reperfusion-induced injury may provide novel pharmacological agents, which can be used in current early reperfusion maneuvring, the established standard therapy for acute myocardial infarction. As no drugs to reduce reperfusion injury are currently available for clinical use $^{8}$ it is interesting to search for new interventions administered at the time of reperfusion.

Bioactive natural products can be considered as very promising molecules for the development of new therapeutic agents and terpenes, which constitute one of the groups with great therapeutic potential. ${ }^{9-12}$ In particular, diterpenes have shown a broad spectrum of biological activities (antibacterial, antiviral, anti-inflammatory, cytotoxic, antitumour, etc), regulating inflammation and the innate immune response. ${ }^{13-17}$ Our previous findings on the antiinflammatory and cytoprotective effects of diterpenes ${ }^{9,10,14}$ prompted us to evaluate a possible therapeutic intervention for cardioprotection (to ameliorate A/R injury). In the present study, we have analyzed targets relevant to the reperfusion injury in an A/R model in cardiomyocytes in order to explore the mechanisms involved in the cardioprotective potential of three labdane diterpenes (Figure 1a). Our data show that two of these diterpenes (T1 and T2) exert significant cardioprotection against $A / R$ injury through inhibition of apoptosis and activation of survival pathways.

\section{Results}

The labdane diterpenes $\mathrm{T} 1$ and $\mathrm{T} 2$ protected $\mathrm{H} 9 \mathrm{c} 2$ cells from A/R-induced apoptosis. We first examined the viability of $\mathrm{H} 9 \mathrm{c} 2$ cells after treatment with diterpenes $(0-100 \mu \mathrm{M})$, using an MTT assay. As seen in Figure 2a, these diterpenes did not significantly affect cell viability at concentrations below $20 \mu \mathrm{M}$, and this dose was selected as the maximal concentration for the subsequent studies. Initial experiments using the experimental protocol shown in

\footnotetext{
'Departamento de Farmacología, Facultad de Farmacia, Universidad Complutense de Madrid, Madrid, Spain and 'Instituto de Investigaciones Biomédicas 'Alberto Sols' (CSIC-UAM), Madrid, Spain

*Corresponding authors: L Boscá, Instituto de Investigaciones Biomédicas 'Alberto Sols' (CSIC-UAM), Arturo Duperier 4, 28029 Madrid, Spain.

Tel: + 349149 72747; Fax: + 349158 54401; E-mail: Ibosca@iib.uam.es

or $\mathrm{B}$ de las Heras, Departamento de Farmacología, Facultad de Farmacia, Universidad Complutense

de Madrid, Plaza Ramón y Cajal s/n, 28040 Madrid, Spain. Tel: + 349139 42276; Fax: + 349139 41726; E-mail: lasheras@farm.ucm.es

Keywords: labdane diterpenes; apoptosis; AKT; ERK; AMPK; cardioprotection

Abbreviations: A/R, anoxia/reperfusion; AMPK, AMP-kinase; DMSO, dimethyl sulfoxide; ATCC, American Type Culture Collection; ERK, extracellular signal regulated-kinase; JNK, c-Jun N-terminal kinase; LDH, lactate dehydrogenase; MAPK, mitogen-activated protein kinase; PI3K, phosphatidylinositol 3-kinase; qRT-PCR, quantitative real-time (RT) polymerase chain reaction (PCR); SDS-PAGE, sodium dodecyl sulphate polyacrylamide gel electophoresis; T1, (dehydroisohispanolone); T2, (8,9-dehydrohispanolone 15,16-lactol); T3, (14,15,16-trisnor-13,9 $\alpha$-hispanolide)

Received 28.7.11; revised 21.9.11; accepted 04.10.11; Edited by RA Knight
} 


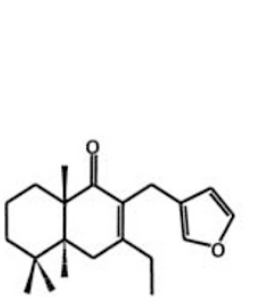

T 1

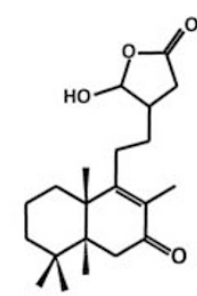

T2

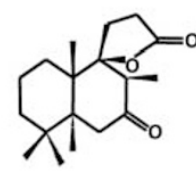

T3
Figure 1 Chemical structure of the labdane diterpenes. Chemical structures of the labdane diterpenes derived from the hispanolone (T1, T2 and T3)

Figure $2 \mathrm{~b}$ were designed to determine the time course in the loss of cell viability after A/R-dependent injury (Figure 2c). $\mathrm{A} / \mathrm{R}$ caused a marked increase of LDH activity in the culture medium, an index of myocyte injury (Figure $2 \mathrm{~d}$ ). Addition of diterpenes $\mathrm{T} 1$ and to a lesser extent of $\mathrm{T} 2$ at $20 \mu \mathrm{M}$ for $4 \mathrm{~h}$ and $6 \mathrm{~h}$ during reoxygenation, significantly reduced the levels of $L D H$ released in response to $A / R$, whereas $T 3$ was inactive. Figure $2 \mathrm{e}$ shows the dose-dependent protection exerted by $T 1$ and $T 2$ at $6 \mathrm{~h}$ after reoxygenation and Figure $2 \mathrm{f}$ shows the loss of protection when the diterpenes were added late after reoxygenation.

The number of apoptotic cells, which were detected as TUNEL-positive nuclei, significantly decreased in cardiomyocytes exposed to $A / R$ and incubated with $T 1$ during reoxygenation ( $6 \mathrm{~h})$, and to a lesser but significant extent with T2 (Figure 2g, left). Agreement was observed between the TUNEL data and the percentage of cells with active caspase-3 as determined by the CaspGlow assay (Figure $2 \mathrm{~g}$, right). Quantitative analysis of these data is shown in Figure $2 \mathrm{~h}$. Similar data were obtained when the effects of the diterpenes T1 and T2 on caspase-3 activity and protein content were determined using specific caspase substrates and antibodies (Figures 2I and J).

T1 and T2 modulated early signaling favoring an antiapoptotic response after $\mathbf{A} / \mathbf{R}$ challenge. We next studied the effects of the diterpenes on proteins involved in survival and apoptotic pathways. In this analysis, expression of proapoptotic proteins such as Bax was markedly increased $6 \mathrm{~h}$ after $A / R$, but remained unchanged in cells treated with $\mathrm{T} 1$ and $\mathrm{T} 2$. In contrast, anti-apoptotic proteins (Bcl-2 and $\mathrm{Bcl}-\mathrm{xL}$ ) exhibited lesser regulation. Moreover, the levels of Hsp70 and the inhibitory protein xIAP also increased after treatment of cells with diterpenes for $6 \mathrm{~h}$ after $A / R$ (Figure $3 \mathrm{a}$ ). The $\mathrm{Bax} / \mathrm{Bcl}-2$ ratio increased in $\mathrm{H} 9 \mathrm{c} 2$ cells after A/R compared with normoxic cells, and this increase was significantly impaired by treatment with $\mathrm{T} 1$ and $\mathrm{T} 2$ (Figure 3a), all these data suggesting that these compounds favor an antiapoptotic profile.

AKT and ERK activation are involved in the protective effects of T1 and T2. Previous results led us to investigate early signaling involved in apoptosis regulation, in particular AKT and MAPKs activities, and the energetic metabolism modulated through AMPK. An initial analysis revealed that both diterpenes induced a time-dependent increase (up to $1 \mathrm{~h}$ ) in the levels of phospho-AKT in cells incubated with T1 and T2 (Figure 3b). Moreover, addition of T1 and T2 after A/R also induced phosphorylation of AKT (both in S473 and T308), an effect suppressed by pretreatment with a PI3K inhibitor (LY294002) and/or AKT inhibitor (Figure 3c). Interestingly, inhibition of the PI3K pathway attenuated the effects of $\mathrm{T} 1$ on $\mathrm{A} / \mathrm{R}$-induced upregulation of Bax and on the active caspase-3 levels. Inhibition of AKT suppressed the protective effects exerted by T1 (Figure 3c). In addition, measurement of caspase-3 activity and cell viability confirmed the relevance of the AKT and to a lesser extent of the PI3K pathways in mediating the effects of T1 after A/R (Figure 3d). MAPKs have been reported to modulate apoptosis in the heart. ${ }^{18,19}$ We therefore examined the activation state of the distinct MAPK pathways to assess their potential involvement in the cardioprotective actions of the diterpenes. A significant basal phosphorylation of ERK1/2 was observed in $\mathrm{H} 9 \mathrm{c} 2$ cells exposed to $A / R$. However, incubation of cells with $\mathrm{T} 1$ and $\mathrm{T} 2$ resulted in a marked activation of ERK phosphorylation after $A / R$, whereas the total protein expression of this kinase remained constant. In contrast, the levels of phospho-p38 were unaffected. As seen in Figure 3e, phospho-JNK was not modulated at the times of sampling. To point out, from an energetic aspect, phospho-AMPK levels were enhanced by diterpenes after $\mathrm{A} / \mathrm{R}$.

When other stimuli apart from $A / R$ were used to induce apoptosis $\left(\mathrm{H}_{2} \mathrm{O}_{2}\right.$, etoposide or staurosporine $)$ the protective effects of diterpenes T1 and T2 were not observed (Figure 4). Minimal protection by $\mathrm{T} 1$ and $\mathrm{T} 2$ was found against $\mathrm{H}_{2} \mathrm{O}_{2}$, but not against etoposide and staurosporine, suggesting a specific protection against $A / R$ injury by these diterpenes.

T1 and T2 protected primary cultures of rat cardiomyocyte against A/R injury. To confirm the cardioprotective effects of diterpenes T1 and T2 observed in H9c2 cells, we moved to primary cultures of rat cardiomyocytes subjected to $A / R$, following the same protocol as above. Addition of T1 and T2 significantly protected cardiomyocytes against A/R-induced injury, as deduced by the decrease in the release of LDH to the culture medium; again, T3 showed a weak activity (Figure 5a). Treatment with T1 and T2 also impaired pro-caspase-3 processing after A/R (Figure $5 b$ ). Expression of Bax following $A / R$ was also attenuated in cells treated with diterpenes, whereas Bcl-2 and xIAP levels remained unaffected (Figure $5 \mathrm{~b}$ ).

The cardioprotective effects exerted by diterpenes in $\mathrm{H} 9 \mathrm{c} 2$ cells involved an increase of phosphorylated AKT, ERK1/2 and AMPK levels that were confirmed in isolated cardiomyocytes (Figures $5 c$ and d). Phospho-p38 levels were moderately increased after A/R regardless of the presence of the diterpenes and phospho-JNK remained dephosphorylated. (Figure 5d).

\section{Discussion}

Protection of the heart from I(A)/R injury still represents a great challenge in the search for new pharmacological agents. $^{20-22}$ Myocardial ischemia is due to blockage of the blood flow in the myocardium, leading to a significant change in the energy balance including depletion of ATP. A 
a

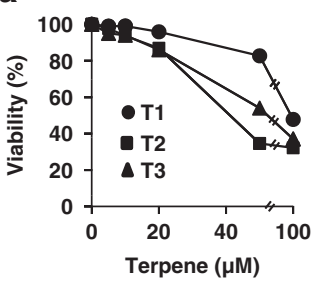

d

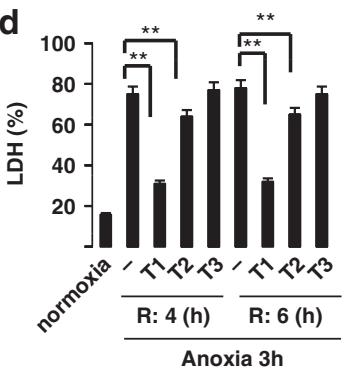

g

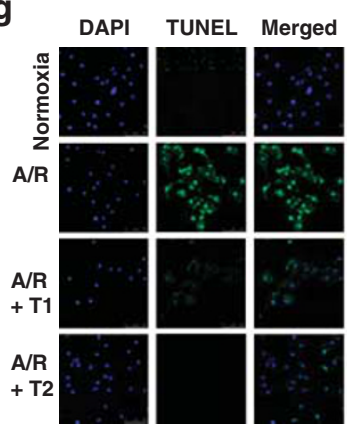

i

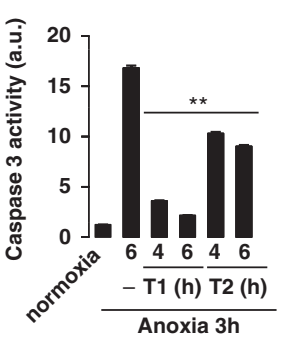

b
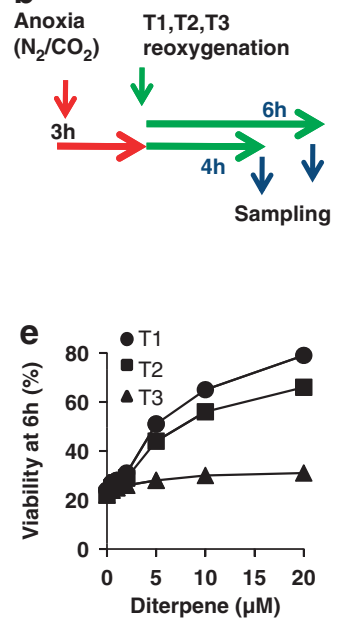

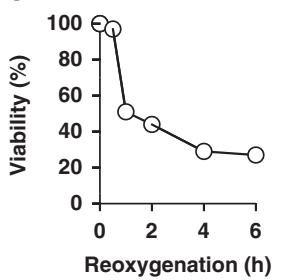

f

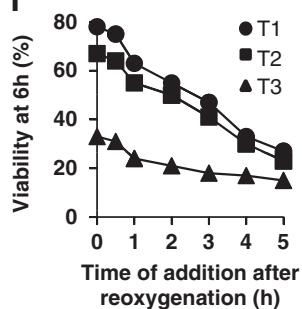

h
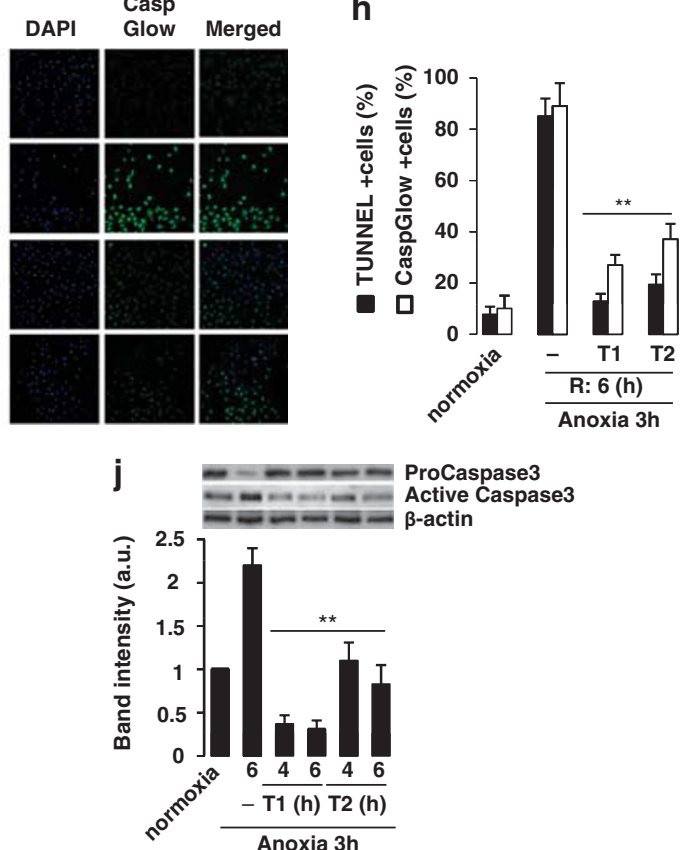

Figure 2 Labdane diterpenes protect H9c2 cells from A/R-induced injury. (a) Viability of H9c2 cells incubated for $24 \mathrm{~h}$ with diterpenes (0-100 $\mu \mathrm{M})$, measured by the MTT assay. (b) Schematic diagram of the experimental protocol (T: diterpenes). (c) Time-dependent effect of reoxygenation on cell viability (MTT assay). (d) LDH release after A/R $(4-6 \mathrm{~h})$. Diterpenes were added prior reoxygenation. $100 \% \mathrm{LDH}$ activity was achieved after addition of $0.5 \%$ Triton X-100 to the cell culture. ${ }^{* *} P<0.01$ treatments versus Triton $\mathrm{X}-100$. (e) Dose-dependent effect of diterpenes on cell viability at $6 \mathrm{~h}$ after A/R. (f) Effect of delayed-addition of diterpenes after A/R. Cell viability was determined by the MTT assay at $6 \mathrm{~h}$ after reoxygenation and expressed as cell viability (\%) versus normoxic cells. (g) TUNEL positive cells (left) and caspase active positive cells (right) expressed as percentage of total cells in diterpene-treated cells versus non-treated cells. ${ }^{*} P<0.01$ compared versus A/R in the absence of diterpene. (h) In situ evaluation of caspase-3 activation. The percentage of caspase-3 positive cells was determined by fluorescence microscopy using the CaspGlow staining kit. (i) Caspase-3 activity and (j) protein levels of pro-caspase-3 and active caspase-3 were determined in cytosolic extracts from cells after A/R and treated with diterpenes T1 and T2. Lane charge was normalized with $\beta$-actin. Western blots were performed in triplicate. Results show the mean \pm S.D. of three experiments. ${ }^{* \star} P<0.01$ treatments versus A/R in the absence of diterpene

compensatory response emerges in the cardiomyocytes from the inhibition of fatty acid oxidation to an increase in the flux toward anaerobic glycolysis. ${ }^{4,5,23,24}$ Although the drop in ATP during ischemia inhibits several basic cell processes, including membrane sodium/potassium-ATPase, reperfusion of the myocardium triggers a rapid increase in intracellular calcium that promotes opening of the mitochondrial permeability transition pore leading to the release of proapoptotic mediators and reactive oxygen species that are key factors in cardiac dysfunction. ${ }^{25}$ In this scenario, we have evaluated the role of labdane diterpenes, members of the bicyclic diterpenoid group, that are potent anti-inflammatory molecules in immune cells and also exhibit cell-protective effects. ${ }^{13}$ In previous studies, we analyzed a series of labdane diterpenes, some of them showing potent anti-inflammatory activity by decreasing the release of inflammatory mediators 
a
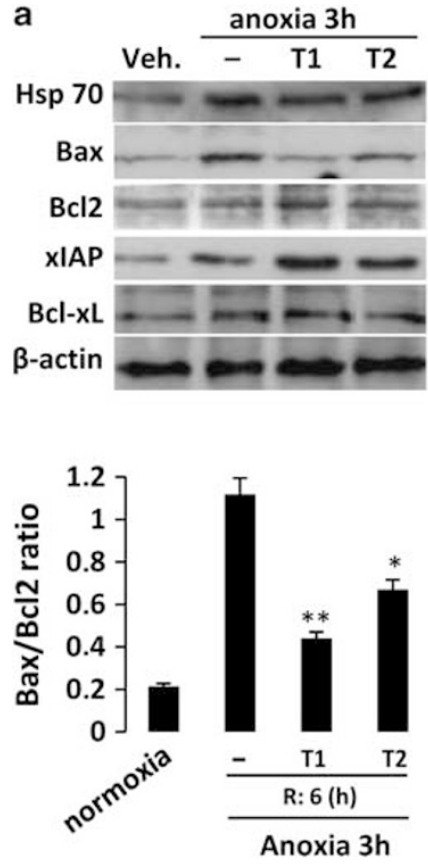

C

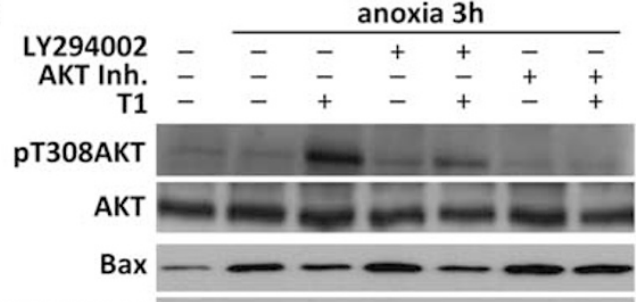

Active casp.3

$\beta$-actin ---

d

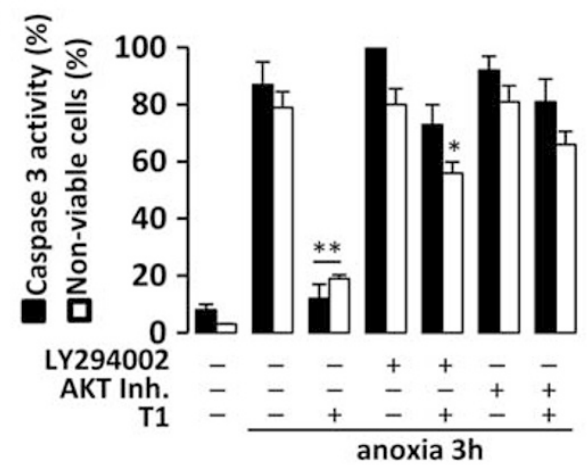

b

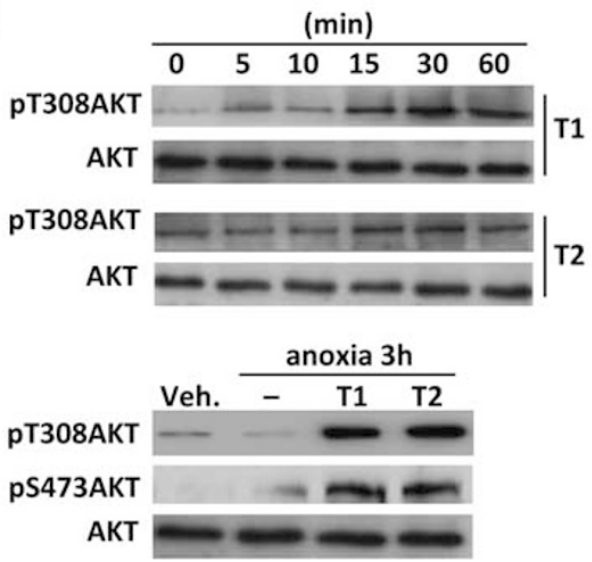

e

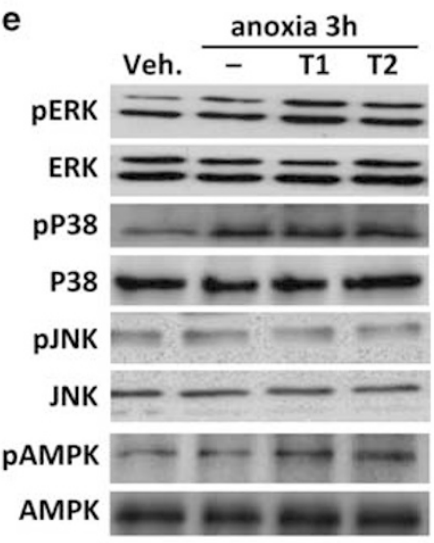

Figure 3 Diterpenes T1 and T2 promote AKT and ERK activation and favor the Bcl-2 family antiapoptotic proteins profile. (a) Levels of Hsp70, Bax, Bcl-2, xIAP and Bcl-xL were determined by western blot from cytosolic extracts from diterpene-treated cells. Protein loading was normalized with $\beta$-actin. The Bax/Bcl-2 ratio was determined at $6 \mathrm{~h}$ after reoxygenation. (b) Time-course of phosphorylation of AKT was determined under normoxic conditions and after A/R. Representative western blots were performed with cytosolic extracts from cells treated with T1 and T2. The samples were immunoblotted and probed with anti-phospho-T308-AKT and anti-phospho-S473-AKT antibodies or anti-AKT antibody as a loading control. (c) Levels of phospho-T308-AKT, Bax and caspase-3 were also determined in the presence or absence of AKT inhibitor, LY294002 and/or T1, using $\beta$-actin as a loading control. (d) Densitometry of caspase-3 activity levels in the presence or absence of AKT inhibitor, LY294002 and/or diterpene T1. (e) Regulation of MAPKs (ERK, p38, JNK) and AMPK. Results show representative blots and the mean \pm S.D. out of three experiments. ${ }^{*} P<0.05$; ${ }^{* \star} P<0.01$ treatments versus $A / R$ in the absence of diterpene

(NO, $\mathrm{PGE}_{2}$, cytokines) through inhibition of NF- $\kappa \mathrm{B}$ activity. ${ }^{14}$ A selection of these diterpenes showing good chemical stability, low toxicity and potent (T2 and T3) or negligible (T3) anti-inflammatory activity have been evaluated in the present work. To our knowledge no other studies have examined the potential of labdane diterpenes for cardioprotection. There are a few that describe a protective role for natural products, such as antioxidant polyphenols, ${ }^{22,26,27}$ and by the triterpene saponins. ${ }^{28,29}$ In the present study, we have focused on the molecular mechanisms underlying the effects of labdanes to protect cardiomyocytes against A/R-induced injury. These diterpenes did not affect cell viability at concentrations lower than $20 \mu \mathrm{M}$, and T1 proved to be safe when used up to $50 \mu \mathrm{M}$ for at least $48 \mathrm{~h}$.

Cardiomyocyte loss after ischemia is mainly due to apoptosis, necrosis (when the cell is unable to support the apoptotic energetic demands), or commitment to autophagy in an attempt to rescue cell function, ${ }^{21,23,30,31}$ all three cellfates having an important role in the heart after I(A)/R-induced dysfunction. Indeed, prevention of the apoptosis induced by ischemia or anoxia minimizes cardiac injury. Our data on early apoptotic signaling after $\mathrm{A} / \mathrm{R}$ shows that $\mathrm{T} 1$ and $\mathrm{T} 2$ protect against cytochrome $c$ release from the mitochondria (not shown), impair Bax accumulation and upregulate the 

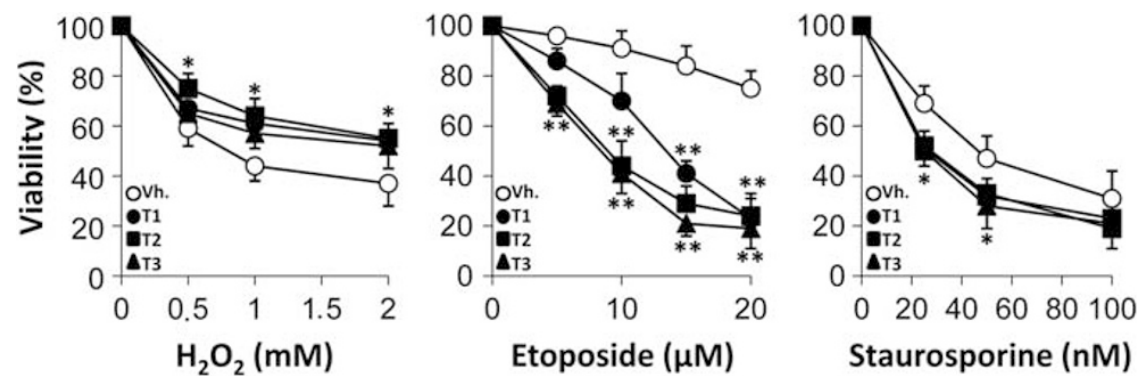

Figure 4 Effect of diterpenes on apoptosis induced by $\mathrm{H}_{2} \mathrm{O}_{2}$, etoposide and staurosporine. Cells were pre-incubated with diterpenes $(20 \mu \mathrm{M})$ for $30 \mathrm{~min}$ and exposed for $18 \mathrm{~h}$ to different concentrations of $\mathrm{H}_{2} \mathrm{O}_{2}$, etoposide, staurosporine or vehicle. Cell viability was evaluated by the MTT assay. Results show the mean \pm S.D. out of four experiments. ${ }^{*} P<0.05 ;{ }^{* *} P<0.01$ treatments versus the vehicle condition

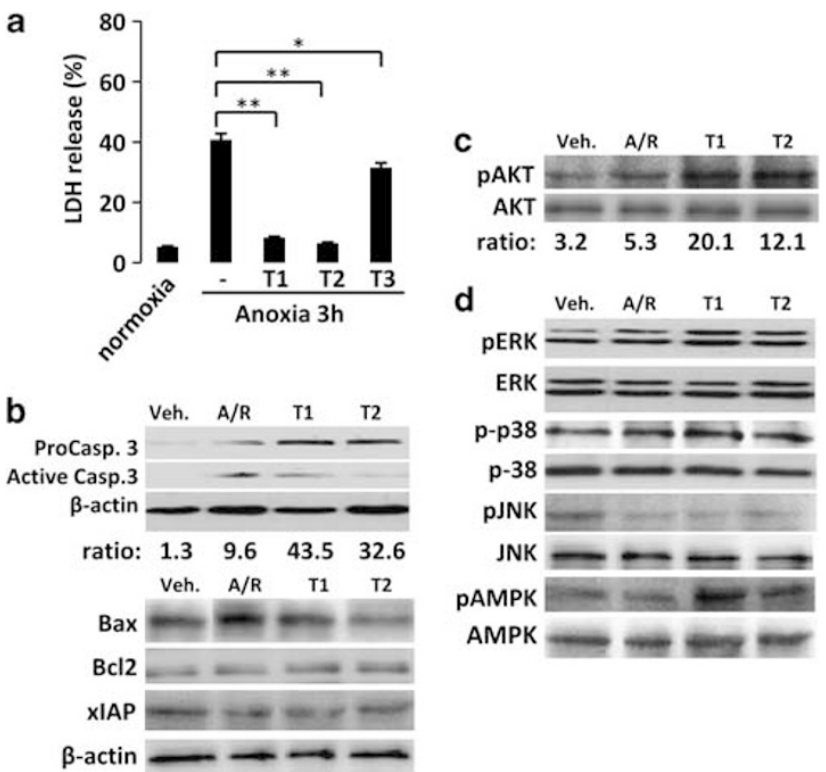

Figure 5 Diterpenes exert cardioprotective effects in isolated rat cardiomyocytes after A/R through inhibition of apoptosis and activation of survival pathways. (a) $\mathrm{LDH}$ release in the supernatants of diterpene-treated rat cardiomyocytes after $\mathrm{A} / \mathrm{R}$ (mean \pm S.D.; $n=4 ;{ }^{*} P<0.05,{ }^{*} \mathrm{P}<0.01$ treatments versus Triton $\mathrm{X}-100$ as $100 \%$ ). Representative western blot analysis ( $n=3-4$ experiments) showing the effects of diterpenes on (b) apoptosis-related proteins (cleaved caspase-3, Bax, $\mathrm{Bcl}-2$ and xIAP), (c) levels of phosphorylated AKT and (d) MAPKs (ERK, p38 and JNK) and AMPK in total extracts obtained from rat cardiomyocytes exposed to A/R at $6 \mathrm{~h}$ after reoxygenation. Western blots were performed in triplicate

levels of the antiapoptotic proteins $x \mid A P, B c l-2$ and $B c l-x L$. In agreement with these data the processing and activity of procaspase- 3 was abrogated after A/R by T1 and T2, and an endpoint of apoptosis, such as TUNEL assays confirmed the protective role of the diterpenes. Interestingly, the protection exerted by T1 and T2 persisted even when these labdanes were administered 1 hour after reoxygenation (less than 15\% reduction in cell viability at $6 \mathrm{~h}$ ) suggesting a significant degree of reversibility in cardiomyocyte injury, when they are added soon after $A / R$.

A large number of studies have shown that PI3K/AKT signaling pathways provide an important cell survival signal for cardiomyocytes..$^{7,32-34}$ In our study, phospho-AKT levels were enhanced by T1 and T2 under basal normoxic conditions, and the phosphorylation in T308 and S473 persisted at least for 1 hour. Under A/R conditions, this phosphorylation was even enhanced and selective inhibition of PI3K or AKT prevented protection by $\mathrm{T} 1$ as deduced by the rise in Bax levels and caspase-3 processing and activation, leading to a drastic loss in cardiomyocyte viability. In addition to this, treatment with $\mathrm{T} 1$ and $\mathrm{T} 2$ also regulated MAPKs phosphorylation in cardiomyocytes exposed to $A / R$, as shown by increased levels of phospho-ERK1/2 (anti-apoptotic) in early reperfusion. However, the levels of p38 that are related to a more proapoptotic condition remained elevated versus the normoxic condition despite the presence of T1 and T2. The levels of phospho-JNK remained unchanged after A/R and minimally decreased when cells were treated with $\mathrm{T} 1$ and T2. Indeed, JNK activation appears to be an important stress kinase in the heart and when activated, can lead to detrimental effects via pro-inflammatory signaling and recruitment of immune cells. ${ }^{19}$ These results were confirmed in rat cardiomyocytes where diterpenes T1 and T2 exerted similar cardioprotective effects through the mentioned survival pathways.

Finally, cardiomyocytes after I(A)/R are facing a metabolic failure and there is a growing body of evidence suggesting that AMPK activation has a relevant cardioprotective function. $^{24,31,35}$ Our data shows a significant activation of AMPK after A/R both in the cardiomyocyte cell line and especially in isolated cardiomyocytes. Although the mechanism governing this process by $\mathrm{T} 1$ is being studied, the data clearly points out to a process activated post anoxia and following reoxygenation, which has potential complementary interventions in this pathway that is under continuous pharmacological progress. In agreement with these observations, transgenic mice expressing a kinase dead form of AMPK that were subjected to low-flow ischemia and reperfusion (I/R) showed significant impairment of glucose uptake, glycolysis and fatty acid oxidation. $^{36}$

Taken together, our data describe an efficient protection of cardiomyocytes by $T 1$ and $T 2$ after $A / R$ and provide new insights to understand the cardioprotective mechanism of labdane diterpenes. Moreover, ischemic heart preconditioning is one of the strategies to improve clinical outcomes in patients with coronary heart disease. The presence of molecules improving cell viability, such as the diterpenes described in this work, deserves additional focus. ${ }^{5}$ In this context, the low cytotoxicity of these compounds in cell culture and their effectiveness in a rat cardiomyocytes $A / R$ model indicates that these labdane diterpenes have potential use in 
the treatment of cardiovascular diseases, and provide useful tools for the development of chemopreventive and chemotherapeutic strategies.

\section{Materials and Methods}

Materials. Diterpenes T1 (dehydroisohispanolone), T2 (8,9-dehydrohispanolone 15,16-lactol) and T3 (14,15,16-trisnor-13,9 $\alpha$-hispanolide) were obtained from hispanolone as previously described. ${ }^{37-39}$

Dulbecco's modified Eagle's medium (DMEM) was purchased from ATCC (American Type Culture Collection). The rest of culture reagents were obtained from Invitrogen (Alcobendas, Spain). Western blot reagents were obtained (purchased)from GE Healthcare (Buckinghamshire, UK). Antibodies were obtained (purchased) from the following suppliers: anti-ERK, anti-pERK, anti-JNK, anti-pJNK, anti-p38, anti-pp38, anti-AKT, anti-pAKT(S-473), anti-pAKT (T-308), anti-AMPK, antipAMPK, anti-caspase-3 (Cell Signaling Technologies, Beverly, MA, USA); antiHsp70 (Gen Script, Piscataway, NJ, USA); anti-Bax, anti-xIAP (BD Biosciences, San Jose, CA, USA); anti-Bcl2, anti-Bcl-xL (Santa Cruz Biotech., Santa Cruz, CA, USA). The fluorescent kit for TUNEL was from Roche (Mannheim, Germany). The CaspGLOW fluorescein active caspase-3 staining kit was obtained (purchased) from BioVision Research (San Francisco, CA, USA) and the specific fluorogenic substrate for caspase-3 was obtained (purchased) from BD Biosciences. Type II collagenase was from Worthington (Lakewood, NJ, USA). LY294002 and kinase inhibitors were obtained (purchased) from Cell Signaling.

Cell culture. H9c2 embryonic rat heart-derived cells were obtained from ATCC. The cells were cultured in DMEM with 10\% fetal bovine serum (FBS), $100 \mathrm{U} / \mathrm{ml}$ penicillin, and $100 \mu \mathrm{g} / \mathrm{ml}$ streptomycin, and maintained in $5 \% \mathrm{CO}_{2}$ at $37^{\circ} \mathrm{C}$. When cell confluence was reached, cells were subcultured by detaching with $0.05 \%$ trypsin-EDTA and reseeding. For experiments, cells were maintained in DMEM plus $2 \%$ FBS.

Isolation of cardiac myocytes was performed as previously described. ${ }^{40}$ Adult mate Wistar rats were heparinized $(4 \mathrm{U} / \mathrm{g})$ and anaesthetized with sodium pentobarbital $(50 \mathrm{mg} / \mathrm{kg})$. The hearts were removed and mounted on a Langerdorffperfusion apparatus. The ascending aorta was cannulated and a retrograde perfusion was set up. The hearts were successively perfused with the following oxygenated solutions at $36^{\circ} \mathrm{C}$ : (1) standard nominally $\mathrm{Ca}^{2+}$-free Tyrode solution (3 min), (2) standard nominally $\mathrm{Ca}^{2+}$-free Tyrode solution ( $15 \mathrm{~min}$ ) containing $60 \mathrm{U} / \mathrm{ml}$ of type II collagenase. The hearts were removed from the Langerdorff apparatus, and after removal of atria, the ventricles were cut off and gently shaken for $3 \mathrm{~min}$ in a standard Tyrode solution containing $0.1 \mathrm{mM} \mathrm{CaCl}_{2}$ to disperse the isolated cells. The resulting cell suspensions were filtered through a $250 \mu$ m nylon mesh and centrifuged for $4 \mathrm{~min}$ at $20 \mathrm{~g}$. Finally, the cell pellets were maintained as the $\mathrm{H} 9 \mathrm{c} 2$ cells in the presence of $0.1 \mathrm{mM} \mathrm{CaCl}_{2}$.

Anoxia/reoxygenation (A/R) protocol. To mimic an $\mathrm{I} / \mathrm{R}$ model (via anoxia in the medium), cells at $80 \%$ confluence were incubated with a glucose-free medium (previously bubbled with $95 \% \mathrm{~N}_{2}$ and $5 \%$ of $\mathrm{CO}_{2}$ ) for $3 \mathrm{~h}$ at $37{ }^{\circ} \mathrm{C}$ in a hypoxic chamber $\left(95 \% \mathrm{~N}_{2}\right.$ and $\left.5 \% \mathrm{CO}_{2}\right)$. For $\mathrm{A} / \mathrm{R}$ studies, the medium was then replaced by maintenance medium (DMEM with $2 \% \mathrm{FBS}$ ) during the reoxygenation period. Diterpenes ( $20 \mu \mathrm{M}$ unless otherwise indicated) were then added to the cells for 4 or $6 \mathrm{~h}$.

Cell viability and apoptosis assays. Cell viability was determined by the 3-[4,5-dimethylthiazol-2-yl]-2,5-diphenyl tetrazolium bromide (MTT) assay following the method described by Mossman. ${ }^{41}$ Cells were seeded at a density of $3 \times 10^{4} \mathrm{cells} /$ well in 96-well plates and were incubated with MTT $(5 \mathrm{mg} / \mathrm{ml})$ for $2 \mathrm{~h}$ at $37^{\circ} \mathrm{C}$. After that, the medium was removed and DMSO $(100 \mu \mathrm{l})$ was added into each well. The optical density (OD) was determined spectrophotometrically at $540 \mathrm{~nm}$.

Lactate dehydrogenase activity (LDH) was determined in the cell culture medium by measuring the conversion of pyruvate to lactate. LDH activity was analyzed spectrophotometrically at $340 \mathrm{~nm}$. The activity of the LDH in the supernatants was compared with the 'total content' in cells disrupted by treatment with $0.5 \%$ Triton X-100.

Caspase-3 activity was spectrofluorometrically determined in cytosolic protein extracts using a specific substrate (Ac-DEVD-AMC) for this caspase, according to the supplier's instructions (BD Biosciences).
In vivo activation of caspase-3 in apoptotic cells was measured by fluorescence microscopy after labeling the cells with the caspase-3 inhibitor DEVD-FMK conjugated to FITC (FITC-DEVD-FMK) as a marker, following the instructions of the supplier of the CaspGLOW fluorescein active caspase-3 staining kit (BioVision Research).

For detection and quantification of apoptosis, the terminal deoxynucleotidyl transferase-mediated dUTP nick end-labeling (TUNEL) assay was performed according to the manufacturer's instructions using a commercially available kit for death detection (Roche). H9c2 cells were seeded at a density of $10^{4}$ cells/well into sterile 8-well Chamber Slides (Falcon, Brookings, SD, USA), exposed to A/R and treated with diterpenes. After reoxygenation $(6 \mathrm{~h})$ cells were fixed with $4 \%$ $p$-formaldehyde for $15 \mathrm{~min}$. Cells were then permeabilized in ice-cold methanol and incubated with $3 \%$ bovine serum albumin (BSA) for $30 \mathrm{~min}$. Coverslips were mounted in Prolong Gold antifade reagent (Invitrogen) and examined using an Espectral Leica TCS SP25 confocal microscope (Leica, Solms, Germany). Alexa fluorescence was excited by the $488 \mathrm{~nm}$ line of an Argon laser 11 and emission collected through a BP filter $(505-530 \mathrm{~nm})$. DAPI fluorescence was excited by the Hg lamp (BP 365/12) and emissions collected through a BP filter (480-520 nm). Values of intensity fluorescence quantification were performed using the Image $\mathrm{J}$ software (NIH, Bethesda, MD, USA).

Protein extraction and western blot. H9c2 cells were washed twice with ice-cold Buffer A (10 mM Hepes, pH 7.9;. 1 mM EDTA, 1 mM EGTA, $10 \mathrm{mM} \mathrm{KCl}$, $1 \mathrm{mM}$ DTT, $0.5 \mathrm{nM}$ PMSF, $2 \mu \mathrm{g} / \mathrm{ml}$ aprotinin, $10 \mu \mathrm{g} / \mathrm{ml}$ leupeptin, $2 \mu \mathrm{g} / \mathrm{ml} \mathrm{TLCK}$ ( $\mathrm{N}_{\alpha}$-tosyl-L-lysine chloromethyl ketone hydrochloride), $5 \mathrm{mM} \mathrm{NaF}, 1 \mathrm{mM} \mathrm{NaVO}{ }_{4}$, $10 \mathrm{mM} \mathrm{Na}_{2} \mathrm{MoO}_{4}$ ) containing $120 \mathrm{mM} \mathrm{NaCl}$ and scraped off the plate. Cells were lysed at $4{ }^{\circ} \mathrm{C}$ with $0.2 \mathrm{ml}$ of buffer A supplemented with $0.5 \%$ Nonidet P-40 with continuous shaking. After centrifugation of the cell lysate the supernatant was stored at $-80^{\circ} \mathrm{C}$ (cytosolic cell extract).

Native cardiomyocytes were homogenized using a hand-held blender in lysis buffer containing (in mM): 50 Tris, 320 sucrose and $1 \mathrm{DTT}, \mathrm{pH} 7.0$, plus a complete protease and phosphatase inhibitors solution. The homogenate was spun at $13000 \mathrm{~g}$ for $10 \mathrm{~min}$ at $4{ }^{\circ} \mathrm{C}$ and supernatants (total cell extract) were frozen and stored at $-80^{\circ} \mathrm{C}$. Protein content in both cases was assayed with the Bio-Rad protein reagent (Bio-Rad, Alcobendas, Spain).

For Western Blot equal amounts of cytosolic protein lysates were separated in $10-15 \%$ sodium dodecyl sulfate-polyacrylamide (SDS-PAGE) gel electrophoresis. The gels were blotted onto a Hybond-P membrane and incubated with the indicated antibodies. Blots were developed by ECL according to the manufacturer's instructions. $\beta$-actin was used as a loading control.

Statistical analysis. All the values are expressed as mean \pm S.D. The statistical significance of differences between the means were determined with the SPSS 19 software (SPSS, Chicago, IL, USA) for Windows using a one-way analysis of variance followed by Bonferroni post-hoc test or Student's $t$-test, as appropriate. A $P$-value of less than 0.05 was considered to be significant.

\section{Conflict of Interest}

The authors declare no conflict of interest.

Acknowledgements. We thank Dr. Benjamín Rodriguez from the Instituto Química Orgánica, CSIC. Madrid (Spain) for kindly providing the diterpenes and Dr. Mercedes Delgado, Instituto Plurisdisciplinar, Universidad Complutense de Madrid for her technical assistance and Ms. Diana Foran, Universidad Complutense de Madrid, for her editorial assistance with the manuscript. This work was supported by grants BFU2011-24760 and PIB2010BZ-00540 from MICINN; red temática de investigación en enfermedades cardiovasculares (RECAVA, RD06/0014/0006), the Instituto de Salud Carlos III (ISCIII), MICINN \& European Regional Development Fund (ERDF) 'Una manera de hacer Europa' and CIBERehd founded by Instituto de Salud Carlos III.

1. Fliss H, Gattinger D. Apoptosis in ischemic and reperfused rat myocardium. Circ Res 1996; 79: 949-956.

2. Ruiz-Meana M, Garcia-Dorado D. Translational cardiovascular medicine (II). Pathophysiology of ischemia-reperfusion injury: new therapeutic options for acute myocardial infarction. Rev Esp Cardiol 2009; 62: 199-209. 
3. Buja LM. Myocardial ischemia and reperfusion injury. Cardiovasc Pathol 2005; 14 $170-175$

4. Hausenloy DJ, Yellon DM. Preconditioning and postconditioning: underlying mechanisms and clinical application. Atherosclerosis 2009; 204: 334-341.

5. Hausenloy DJ, Yellon DM. The therapeutic potential of ischemic conditioning: an update. Nat Rev Cardiol 2011; 6: 12-13.

6. Zhu M, Feng J, Lucchinetti E, Fischer G, Xu L et al. Ischemic postconditioning protects remodeled myocardium via the PI3K-PKB/Akt reperfusion injury salvage kinase pathway. Cardiovasc Res 2006; 72: 152-162.

7. Hausenloy DJ, Yellon DM. New directions for protecting the heart against ischaemiareperfusion injury: targeting the Reperfusion Injury Salvage Kinase (RISK)-pathway. Cardiovasc Res 2004; 61: 448-460.

8. Downey JM, Cohen MV. Why do we still not have cardioprotective drugs? Circ J 2009; 73 $1171-1177$.

9. de las Heras B, Hortelano S, Giron N, Bermejo P, Rodriguez B et al. Kaurane diterpenes protect against apoptosis and inhibition of phagocytosis in activated macrophages. Br J Pharmacol 2007; 152: 249-255.

10. Castrillo A, de las Heras B, Hortelano S, Rodriguez B, Villar A et al. Inhibition of the nuclear factor $\kappa \mathrm{B}$ pathway by tetracyclic kaurene diterpenes in macrophages. Specific effects on $\mathrm{NF}-\kappa \mathrm{B}$-inducing kinase activity and on the coordinate activation of ERK and p38 MAPK J Biol Chem 2001; 276: 15854-15860.

11. de las Heras $B$, Hortelano $S$. Molecular basis of the anti-inflammatory effects of terpenoids. Inflamm Allergy Drug Targets 2009; 8: 28-39.

12. Gurusamy N, Lekli I, Mukherjee S, Ray D, Ahsan MK et al. Cardioprotection by resveratrol: a novel mechanism via autophagy involving the mTORC2 pathway. Cardiovasc Res 2010; 86: 103-112.

13. Chinou I. Labdanes of natural origin-biological activities (1981-2004). Curr Med Chem 2005; 12: 1295-1317.

14. Giron N, Traves PG, Rodriguez B, Lopez-Fontal R, Bosca L et al. Suppression of inflammatory responses by labdane-type diterpenoids. Toxicol Appl Pharmacol 2008; 228 179-189.

15. Dong Y, Morris-Natschke SL, Lee KH. Biosynthesis, total syntheses, and antitumor activity of tanshinones and their analogs as potential therapeutic agents. Nat Prod Rep 2011; 28 : 529-542.

16. Navarro A, de las Heras B, Villar AM. Andalusol, a diterpenoid with anti-inflammatory activity from Siderits foetens Clemen. Z Naturforsch C 1997; 52: 844-849.

17. de las Heras B, Navarro A, Diaz-Guerra MJ, Bermejo $P$, Castrillo $A$ et al. Inhibition of NOS-2 expression in macrophages through the inactivation of NF- $\kappa$ B by andalusol. Br J Pharmacol 1999; 128: 605-612.

18. Ren J, Zhang S, Kovacs A, Wang Y, Muslin AJ. Role of p38 $\alpha$ MAPK in cardiac apoptosis and remodeling after myocardial infarction. J Mol Cell Cardiol 2005; 38: 617-623.

19. Qi D, Hu X, Wu X, Merk M, Leng $L$ et al. Cardiac macrophage migration inhibitory facto inhibits JNK pathway activation and injury during ischemia/reperfusion. J Clin Invest 2009; 119: 3807-3816.

20. Mann DL. The emerging role of innate immunity in the heart and vascular system: for whom the cell tolls. Circ Res 2011; 108: 1133-1145.

21. Singh SS, Kang PM. Mechanisms and Inhibitors of Apoptosis in Cardiovascular Diseases. Curr Pharm Des 2011; 17: 1783-1793.

22. Zhao $Y$, Zhao B. Protective effect of natural antioxidants on heart against ischemiareperfusion damage. Curr Pharm Biotechnol 2010; 11: 868-874.

23. Turer AT, Hill JA. Pathogenesis of myocardial ischemia-reperfusion injury and rationale for therapy. Am J Cardiol 2010; 106: 360-368.

24. Wang W, Lopaschuk GD. Metabolic therapy for the treatment of ischemic heart disease: reality and expectations. Expert Rev Cardiovasc Ther 2007; 5: 1123-1134.
25. Carreira RS, Lee P, Gottlieb RA. Mitochondrial therapeutics for cardioprotection. Curr Pharm Des 2011; 17: 2017-2035.

26. Thomas CJ, Ng DC, Patsikatheodorou N, Limengka Y, Lee MW et al. Cardioprotection from ischaemia-reperfusion injury by a novel flavonol that reduces activation of p38 MAPK. Eur J Pharmacol 2011; 658: 160-167.

27. Townsend PA, Scarabelli TM, Pasini E, Gitti G, Menegazzi M et al. Epigallocatechin-3gallate inhibits STAT-1 activation and protects cardiac myocytes from ischemia/ reperfusion-induced apoptosis. FASEB J 2004; 18: 1621-1623.

28. Tsutsumi YM, Tsutsumi R, Mawatari K, Nakaya Y, Kinoshita M et al. Compound K, a metabolite of ginsenosides, induces cardiac protection mediated nitric oxide via Akt/PI3K pathway. Life Sci 2011; 88: 725-729.

29. Li C, Tian J, Li G, Jiang W, Xing Y et al. Asperosaponin VI protects cardiac myocytes from hypoxia-induced apoptosis via activation of the PI3K/Akt and CREB pathways. Eur J Pharmacol 2010; 649: 100-107.

30. Matsui $\mathrm{Y}$, Takagi $\mathrm{H}, \mathrm{Qu} X$, Abdellatif M, Sakoda $\mathrm{H}$ et al. Distinct roles of autophagy in the heart during ischemia and reperfusion: roles of AMP-activated protein kinase and Beclin 1 in mediating autophagy. Circ Res 2007; 100: 914-922.

31. Takagi H, Matsui Y, Hirotani S, Sakoda H, Asano T et al. AMPK mediates autophagy during myocardial ischemia in vivo. Autophagy 2007; 3: 405-407.

32. Wang Y. Mitogen-activated protein kinases in heart development and diseases. Circulation 2007; 116: 1413-1423.

33. Kupatt C, Hinkel R, Lamparter M, von Bruhl ML, Pohl T et al. Retroinfusion of embryonic endothelial progenitor cells attenuates ischemia-reperfusion injury in pigs: role of phosphatidylinositol 3-kinase/AKT kinase. Circulation 2005; 112: 117-122.

34. Matsui T, Tao J, del Monte F, Lee KH, Li L et al. Akt activation preserves cardiac function and prevents injury after transient cardiac ischemia in vivo. Circulation 2001; 104: 330-335.

35. Wang Y, Gao E, Tao L, Lau WB, Yuan Y et al. AMP-activated protein kinase deficiency enhances myocardial ischemia/reperfusion injury but has minimal effect on the antioxidant/ antinitrative protection of adiponectin. Circulation 2009; 119: 835-844.

36. Russell 3rd RR, Li J, Coven DL, Pypaert M, Zechner C et al. AMP-activated protein kinase mediates ischemic glucose uptake and prevents postischemic cardiac dysfunction, apoptosis, and injury. J Clin Invest 2004; 114: 495-503.

37. García-Álvarez M, Pérez-Sirvent L, Rodriguez B. Transformaciones de hispanolona I. Síntesis parcial del diterpenoide furolabdánico galeopsinas. Anales Quím 1981; 77: 316-319.

38. Pérez-Sirvent L, García-Álvarez MC, Rodríguez B. Transformaciones de hispanolona II. Reacción retroaldólica de hispanolona y condensación aldólica de $\delta$-dicetonas diterpénicas. Anales Quím 1981; 77: 324-329.

39. Pérez-Sirvent L, García-Álvarez MC, Balestrieri MA, Rodríguez B. Transformaciones de hispanolona III. Reacciones del anillo B y degradación a di-homo-drimanos. Anales Quím 1981; 77 : 330-334

40. Fernandez-Velasco M, Goren N, Benito G, Blanco-Rivero J, Bosca L et al. Regional distribution of hyperpolarization-activated current (If) and hyperpolarization-activated cyclic nucleotide-gated channel mRNA expression in ventricular cells from control and hypertrophied rat hearts. J Physiol 2003; 553: 395-405.

41. Mossman T. Rapid colorimetric assay for cellular growth and survivals: application to proliferation and cytotoxicity assays. J Immunol Methods 1983: 55-63.

Cell Death and Disease is an open-access journal published by Nature Publishing Group. This work is licensed under the Creative Commons Attribution-Noncommercial-Share Alike 3.0 Unported License. To view a copy of this license, visit http:// creativecommons.org/licenses/by-nc-sa/3.0/ 